\title{
Conhecimento de puérperas sobre analgesia do parto normal em maternidade pública no interior do Rio Grande do Norte, Brasil
}

Knowledge of puerperas about analgesia of normal delivery in public maternity in the interior of Rio Grande do Norte, Brazil

Conocimiento de puerperas sobre analgesia de entrega normal en maternidad pública en el interior de Rio Grande do Norte, Brasil

Rachel de Alcântara Oliveira Ramalho

ORCID: https://orcid.org/0000-0002-4907-9675 Universidade Federal do Rio Grande do Norte, Brasil E-mail: rachelramalho@msn.com

Rilva Lopes Sousa Muñoz

ORCID: https://orcid.org/0000-0001-6949-5775 Universidade Federal da Paraíba, Brasil E-mail: rilvamunoz@gmail.com

\begin{abstract}
Resumo
Objetivo: Avaliar o conhecimento de puérperas sobre a analgesia de parto vaginal, e a associação entre a existência desta informação e a decisão e o desfecho quanto ao tipo de parto no Hospital Universitário Ana Bezerra (HUAB), em Santa Cruz, Rio Grande do Norte, Brasil. Metodologia: O modelo deste estudo foi descritivo e transversal, de abordagem quantitativa. A amostra foi composta por puérperas atendidas na maternidade do HUAB. Os dados foram coletados por meio de entrevistas diretas, com preenchimento de questionário estruturado, assim como revisão de prontuários. Resultados: Foram entrevistadas 99 puérperas. Observou-se que 78,8\% tiveram parto normal e houve relação estatisticamente significativa deste tipo de parto normal com maior nível de escolaridade, renda maior que um salário-mínimo e trabalho remunerado. Verificou-se que apenas $22,2 \%$ sabiam da disponibilidade da analgesia para o parto vaginal. A decisão da gestante sobre o tipo de parto também se associou com a ocorrência de parto vaginal, mas não houve associação entre conhecimento da existência de analgesia do parto na maternidade com a decisão materna ou com a realização do parto vaginal. Conclusão: $O$ grau de conhecimento sobre disponibilidade de analgesia para o parto foi baixo, compatível com resultados de outros países em desenvolvimento. O conhecimento materno sobre a disponibilidade de alívio da dor do parto se relacionou com nível de escolaridade, renda e trabalho remunerado, mas não se associou com a decisão materna pelo parto vaginal ou com a realização deste tipo de parturição.
\end{abstract}

Palavras-chave: Trabalho de parto; Analgesia obstétrica; Maternidade hospitalar.

\begin{abstract}
Objective: To assess the knowledge of postpartum women about analgesia in vaginal labor, as well as the association between the existence of this information and the decision and outcome regarding the type of delivery in Ana Bezerra University Hospital (HUAB), in Santa Cruz, Rio Grande do Norte, Brazil. Methodology: The model of this study was descriptive and cross-sectional, with a quantitative approach. The sample consisted of puerperal women attended at the HUAB maternity hospital. Data were collected through direct interviews, with the completion of a structured questionnaire, as well as a review of medical records. Results: 99 puerperal women were interviewed. It was observed that $78.8 \%$ had vaginal delivery and there was a statistically significant relationship between this type of vaginal delivery and higher education level, income higher than the minimum wage and paid work. It was found that only $22.2 \%$ knew about the availability of analgesia for vaginal delivery. The pregnant woman's decision about the type of delivery was also associated with the occurrence of vaginal delivery, but there was no association between knowledge of the existence of analgesia during labor in the maternity ward with the mother's decision or with the performance of vaginal delivery. Conclusion: The rate of knowledge about the availability of analgesia for childbirth was low, consistent with results from other developing countries. Maternal knowledge about the availability of pain relief during labor was related to education level, income, and paid work, but was not associated with the maternal decision for vaginal delivery or with this type of parturition.
\end{abstract}

Keywords: Labor; Obstetric analgesia; Hospital maternity.

\section{Resumen}

Objetivo: Evaluar el conocimiento de las puérperas sobre la analgesia en el trabajo de parto vaginal, así como la asociación entre la existencia de esta información y la decisión y resultado respecto al tipo de parto en el Hospital Universitario Ana 
Bezerra (HUAB). en Santa Cruz, Rio Grande do Norte, Brasil. Metodología: El modelo de este estudio fue descriptivo y transversal, con enfoque cuantitativo. La muestra estuvo constituida por puérperas atendidas en la maternidad del HUAB. Los datos fueron recolectados a través de entrevistas directas, con la cumplimentación de un cuestionario estructurado, así como una revisión de historias clínicas. Resultados: Se entrevistaron 99 puérperas. Se observó que el 78,8\% tuvo parto vaginal y existió una relación estadísticamente significativa entre este tipo de parto vaginal y nivel educativo superior, ingresos superiores al salario mínimo y trabajo remunerado. Se encontró que solo el 22,2\% conocía la disponibilidad de analgesia para el parto vaginal. La decisión de la gestante sobre el tipo de parto también se asoció con la ocurrencia de parto vaginal, pero no hubo asociación entre el conocimiento de la existencia de analgesia durante el trabajo de parto en la sala de maternidad con la decisión de la madre o con la realización de parto vaginal. Conclusión: El nivel de conocimiento sobre la disponibilidad de analgesia para el parto fue baja, consistente con los resultados de otros países en desarrollo. El conocimiento materno sobre la disponibilidad de alivio del dolor durante el trabajo de parto se relacionó con el nivel de educación, los ingresos y el trabajo remunerado, pero no se asoció con la decisión materna de parto vaginal o con este tipo de parto.

Palabras-clave: Trabajo de parto; Analgesia obstétrica; Maternidad hospitalaria.

\section{Introdução}

A realização das boas práticas de atenção ao parto e o bem-estar da parturiente compõem a quinta meta dos Objetivos de Desenvolvimento Sustentável da Organização das Nações Unidas, a Agenda 2030 (United Nations, 2016). Nesse sentido, a tecnologia e a humanização do parto não são fatores excludentes entre si. Existem várias maneiras de garantir o conforto com suporte durante o trabalho de parto no ambiente hospitalar. A humanização da assistência ao parto vaginal também inclui o trabalho do anestesiologista como elemento fundamental do atendimento à parturiente, se este for o desejo desta, resgatando sua autonomia, em práticas comprovadamente benéficas na parturição (Freitas \& Meinberg, 2009).

A analgesia obstétrica não é uma prática comum nos partos vaginais e muitas vezes é negligenciada, ainda que a dor associada às contrações uterinas possa ser aliviada pelo uso de técnicas farmacológicas e não farmacológicas, ou pela combinação destas (Endalew et al., 2020). Contudo, em regiões onde predomina baixo nível socioeconômico da população, como no Nordeste do Brasil, a escassez de conhecimento por parte das gestantes, atitudes negativas em relação à analgesia farmacológica, falta de pessoal treinado e ausência de protocolos clínicos estabelecidos nos serviços podem constituir impedimentos ao adequado uso de analgesia durante o parto normal (Brasil, 2017).

A falta de conhecimento pelas gestantes sobre a disponibilidade de analgesia obstétrica é uma razão importante para sua falta de utilização rotineira (Workie et al., 2021; Nabukenya et al., 2015). Por outro lado, estudos evidenciam que muitas vezes os partos cesarianos decorrem da solicitação materna em virtude de experiências anteriores com a dor do parto vaginal (Prakash et al., 2017; Shaaban et al., 2017). A analgesia farmacológica do parto difundiu-se em muitos centros, alcançando taxas acima de 50\%, particularmente mais elevada em primigestas, havendo, paralelamente, diminuição das taxas de cesarianas eletivas, o que provavelmente reflete aspectos psicoemocionais das parturientes em relação ao enfrentamento da dor (Leão, 2016). É imprescindível a qualificação da atenção à gestante no sentido de garantir que a decisão pela via de parto considere os ganhos em saúde e seus possíveis riscos, de maneira informada e compartilhada por ela com a equipe de saúde que a atende.

A principal pergunta desta pesquisa é a seguinte: O conhecimento sobre a disponibilidade de analgesia do trabalho de parto vaginal associa-se à escolha materna do tipo de parto? A principal hipótese, como resposta provisória a esta pergunta, é a de que o conhecimento pela gestante acerca da disponibilidade de alívio farmacológico da dor durante o trabalho de parto normal associa-se a uma menor frequência de decisões para realização de cesarianas, em comparação com a ausência de conhecimento prévio sobre disponibilidade do alívio da dor.

$\mathrm{O}$ objetivo deste estudo foi avaliar o conhecimento de puérperas em relação à analgesia farmacológica durante o trabalho de parto vaginal em uma maternidade pública que pactua com programas de humanização do atendimento obstétrico, assim como a associação entre a existência da informação e a decisão quanto ao tipo de parto realizado na maternidade do Hospital Universitário Ana Bezerra, em Santa Cruz, Rio Grande do Norte (RN), Brasil. 


\section{Metodologia}

O modelo deste estudo foi descritivo e transversal, de abordagem quantitativa. Um estudo descritivo consiste em uma pesquisa empírica cuja principal finalidade é a descrição de características de fenômenos por meio de entrevistas e/ou questionários (Marconi \& Lakatos, 2003), com o objetivo de analisar relacionamentos entre variáveis, sem buscar conexão de causa-efeito (Sousa et al., 2007).

A amostra foi composta por puérperas atendidas na maternidade pública do Hospital Universitário Ana Bezerra (HUAB), localizado no centro da cidade de Santa Cruz-RN, município-polo em relação às demais cidades da Mesorregião do Agreste Potiguar, na microrregião do Trairi. O município de Santa Cruz está a 115 quilômetros de distância da capital do RN, Natal. A população desta cidade tem cerca de 39.355 habitantes (IBGE, 2016). No referido município, existe apenas este serviço de assistência ao parto, a maternidade do HUAB, referência para atendimento obstétrico e pediátrico no território do Trairi.

Na maternidade do HUAB foram realizados mensalmente, em média, 235,2 partos no ano de 2019, segundo dados verificados no livro de registro de partos do serviço. O HUAB é um hospital onde se realizam atividades de ensino, pesquisa e extensão, com 100\% dos leitos destinados aos usuários do Sistema Único de Saúde (SUS), com atendimento ambulatorial, de internamento, de urgência e emergência (Brasil, 2019).

Mediante amostragem não-probabilística por conveniência, foram incluídas na pesquisa puérperas atendidas em um período de seis meses na maternidade. Foram consideradas elegíveis puérperas assistidas na maternidade do HUAB para realização do parto a termo entre os meses de agosto de 2020 e fevereiro de 2021, independentemente de a via de parto ter sido vaginal ou cesariana. Foram excluídas do estudo as puérperas nas seguintes condições: (a) com diagnóstico de gestação de alto risco ou presença de condição clínica que por si só representasse indicação para parto cesariano; (b) partos de natimortos; (c) gestações gemelares; (d) admissão hospitalar em período expulsivo e/ou que tiveram partos fora das unidades da maternidade em questão (carro, casa, ambulância); (e) falta de compreensão do idioma (português) pelas puérperas; e (h) transtornos mentais graves.

A variável primária do estudo foi mensurada como uma variável autorreferida e nominal, a partir das respostas ("sim", "não", "não sabe responder") à pergunta se alguma vez as participantes foram informadas (pelo seu médico, outro profissional de saúde, ou por outras fontes) sobre o uso de analgesia para o parto normal. Considerou-se que as participantes tinham conhecimento sobre a existência de analgesia do trabalho de parto quando elas responderam afirmativamente ao serem questionadas por meio da referida pergunta, independentemente da fonte de informação. As variáveis secundárias foram tipo de parto (vaginal ou cesariano) e as variáveis sociodemográficas. Foi considerada como assistência pré-natal completa quando a gestante realizou mais de seis consultas (Brasil, 2006).

Os dados foram coletados por meio de entrevistas diretas e estruturadas, com base em instrumento elaborado especificamente para este fim. Os itens incluídos no questionário foram escolhidos a partir de dados de investigações publicadas anteriormente (Monte, 2013; Costa et al., 2004), após adaptação aos objetivos do presente estudo, assim como com adição de outros itens elaborados primariamente pelas pesquisadoras. O questionário foi composto de perguntas fechadas do tipo dicotômico e de múltipla escolha. Houve itens em que foram fornecidas opções pré-selecionadas e as entrevistadas poderiam escolher a opção "outra" para fornecer resposta livre. Na primeira parte do questionário, foram registrados dados sociodemográficos (idade, escolaridade, raça/cor, renda, estado civil, ocupação remunerada), tipo de parto e se a decisão sobre o tipo de parto foi compartilhada (médico-paciente), indicação médica ou decisão das participantes. Para efeito de análise, a variável raça/cor da pele foi autorrelatada, considerando-se as categorias branca, negra, parda e indígena.

$\mathrm{Na}$ segunda parte do questionário, foram registrados dados sobre o conhecimento materno em relação ao alívio farmacológico da dor no trabalho de parto e a disponibilidade deste tipo de analgesia para o parto normal na maternidade, fonte 
da informação recebida, experiência da intensidade da dor, administração de analgesia da dor durante o trabalho de parto e desejo pessoal em relação ao alívio da dor e a decisão (pessoal, médica, compartilhada) na escolha do tipo de parto na maternidade, assim como dados relacionados à gravidez. Uma escala visual analógica (EVA) foi utilizada para avaliar a percepção das gestantes em relação à intensidade da dor relacionada ao processo de trabalho de parto (expectativa e experiência vivenciada).

A aplicação do questionário foi presencial, em ambiente da maternidade, no Alojamento Conjunto, ou outra sala do serviço, mas proporcionando privacidade às respondentes, de 12 a 24 horas após o parto, nos expedientes da manhã ou da tarde, em qualquer dia da semana, conforme funcionamento do serviço. Informações sobre o trabalho de parto e registros a respeito da assistência ao parto foram pesquisadas nos prontuários.

A variável primária foi mensurada como autorreferida e nominal, a partir das respostas ("sim", "não", "não sabe") à pergunta se as participantes sabiam da existência de analgesia farmacológica do parto normal. A esta variável se atribuiu o termo "conhecimento" no sentido de noção ou conhecimento imediato, elementar ou superficial. Associada a esta questão, perguntou-se se elas foram informadas sobre o uso de analgesia para o parto durante a gestação e caso afirmativo, sua fonte (pelo seu médico, outro profissional de saúde e qual, amigos, familiares, televisão, internet, ou outra fonte). As variáveis secundárias foram tipo de parto (dicotômica: vaginal ou normal; cesariano) e variáveis sociodemográficas.

Os entrevistadores foram médicos residentes de Anestesiologia em atividade no HUAB e submetidos a treinamento prático prévio com a pesquisadora responsável, incluindo técnica de entrevista e questões relacionadas ao preenchimento do instrumento de pesquisa. Embora o questionário fosse um instrumento autoaplicável, este foi administrado pelos entrevistadores, que fizeram as perguntas verbalmente às puérperas, face a face, lendo as questões e anotando suas respostas no papel. As únicas instruções dadas foram sobre a atenção a todas as questões, assim como sobre não haver respostas certas ou erradas e da necessidade de respostas que correspondessem com a maior exatidão possível ao caso pessoal.

A análise dos dados foi baseada em estatística descritiva, com determinação de estimadores qualitativos e quantitativos, assim como análise inferencial não-paramétrica para avaliar associações entre variáveis categóricas, por meio do teste qui-quadrado de Pearson, ou do teste exato de Fisher, quando apropriado. As respostas aos itens dos questionários foram codificadas de acordo com o nível de mensuração nominal e ordinal e mostrados por meio de representação tabular e gráfica. $\mathrm{Na}$ análise inferencial, foram considerados estatisticamente significantes valores de $p$ menores que $5 \%$. Foram avaliadas as relações entre as respostas aos itens dos questionários e as variáveis sociodemográficas, incluindo faixa de idade, estado civil, raça/etnia, ocupação remunerada, renda e escolaridade.

Visando atender ao objetivo do estudo, foram traçadas as seguintes hipóteses estatísticas:

- H0: o conhecimento materno sobre a disponibilidade de alívio medicamentoso da dor durante o trabalho de parto não é baixo e não se associa com a ocorrência de parto cesariano; e

- H1: o conhecimento materno sobre a disponibilidade de alívio medicamentoso da dor durante o trabalho de parto é baixo e se associa com a ocorrência de parto cesariano.

Para a análise estatística, foi utilizado o programa informático Statistical Package for the Social Sciences (SPSS) na versão 21.0 para o sistema operativo Windows (IBM Corporation, Armonk, NY, EUA).

Conforme a Resolução 466/12 do Conselho Nacional de Saúde para Pesquisas Envolvendo Seres Humanos, este projeto foi aprovado pelo Comitê de Ética em Pesquisa do Centro de Ciências Médicas da UFPB sob Parecer no 3.944.430.

\section{Resultados}

A análise do perfil sociodemográfico das 99 puérperas entrevistadas mostrou que a idade variou entre 14 e 36 anos (média: 24,8; desvio-padrão: 5,6), observando-se que 64,6\% da amostra eram adultas jovens (entre 20 e 40 anos) e 30,3\% eram 
adolescentes (14 a 19 anos). A grande maioria encontrava-se casada ou em união consensual (87,9\%) e 68,7\% se consideraram pardas. No que se refere à escolaridade, 49,5\% tinham o ensino médio completo. Quanto à renda, 52,5\% viviam com até um salário-mínimo e 48,5\% tinham ocupação remunerada. As ocupações mais frequentemente referidas foram relacionadas ao trabalho no comércio, bancos, transportes e hotelaria $(25,3 \%)$ e à agricultura $(12,1 \%)$.

$\mathrm{Na}$ caracterização de gestações anteriores, realização de pré-natal e tipo de parto, 62,6\% das entrevistadas afirmaram que aquela não havia sido sua primeira gravidez/parto, e todas haviam realizado pré-natal, 78,8\% com assistência pré-natal completa, ou seja, com mais de seis consultas. Em relação ao parto, a maioria teve parto vaginal $(78,8 \%)$. Portanto, a prevalência de parto cesariano foi de $21,2 \%$. Observou-se que $37,4 \%$ afirmaram que a decisão quanto ao tipo de parto foi compartilhada com o médico, enquanto 79,8\% estavam em trabalho de parto ao chegarem à maternidade. Quando questionadas sobre a via de parto preferida por elas, 78,8\% responderam que havia sido o parto vaginal. Em relação à via de parto indicada pelos seus médicos obstetras, as participantes responderam que $86,9 \%$ também indicaram parto vaginal.

$\mathrm{Na}$ caracterização da dor do trabalho de parto e informações sobre analgesia, em uma escala de 0 a 10, a média de dor que as entrevistadas referiram foi de 9,2 (desvio-padrão: 1,7). Além disso, a principal preocupação em relação ao parto foi a intensidade da dor para 55,6\% das participantes, porém se fosse oferecido um medicamento para aliviar a dor, 94,9\% delas o aceitariam. Por outro lado, 77,8 \% das puérperas afirmaram que a dor que sentiram correspondeu às suas expectativas. Durante o trabalho de parto, $71,7 \%$ das parturientes afirmaram que receberam alguma técnica de alívio da dor e as mais citadas foram banho de chuveiro, bola, deambulação e compressas. Nenhuma das participantes do estudo recebeu analgesia farmacológica.

Observou-se que $62,6 \%$ das entrevistadas afirmaram que aquela não havia sido sua primeira gravidez/parto. Todas haviam realizado pré-natal, sendo 78,8\% com assistência pré-natal completa (mais de seis consultas). Em relação ao parto, a maioria teve parto normal (78,8\%); assim, a prevalência de parto cesariano na amostra foi de $21,2 \%$. Observou-se que $79,8 \%$ estavam em trabalho de parto ao chegarem à maternidade. Quando questionadas sobre a via de parto preferida por elas, $78,8 \%$ responderam que seu desejo era pelo parto vaginal. Em relação à via de parto indicada pelos médicos que as acompanhavam, $86,9 \%$ referiram que a decisão obstétrica também foi pelo parto por via vaginal. No que concerne ao compartilhamento sobre a decisão quanto ao tipo de parto, $68,7 \%$ das entrevistadas relataram terem se sentido incluídas na decisão e 48,5\% julgaram ter recebido informações adequadas sobre o que estava sendo planejado e o que aconteceria durante o trabalho de parto.

Quando questionadas especificamente se haviam desejado o alívio da dor do parto, 63,6\% responderam que sim, mas apenas 22,2 sabiam que existia disponibilidade de medicação para analgesia deste tipo de parto na maternidade. Em relação às informações gerais sobre o trabalho de parto, $88,9 \%$ afirmaram que receberam alguma informação durante a gestação. Das mulheres que manifestaram desejo de receber alívio da dor durante o parto, apenas 59 afirmaram ter recebido alguma orientação sobre o assunto no período do pré-natal e que a principal fonte desta informação foi fornecida pelo enfermeiro da equipe de saúde (Figura 1). 
Figura 1 Fontes de informação sobre analgesia do trabalho de parto vaginal de puérperas que desejaram ter recebido analgesia por medicamentos na maternidade do Hospital Universitário Ana Bezerra, Santa Cruz-RN entre agosto de 2020 e fevereiro de $2021(\mathrm{n}=59)$.

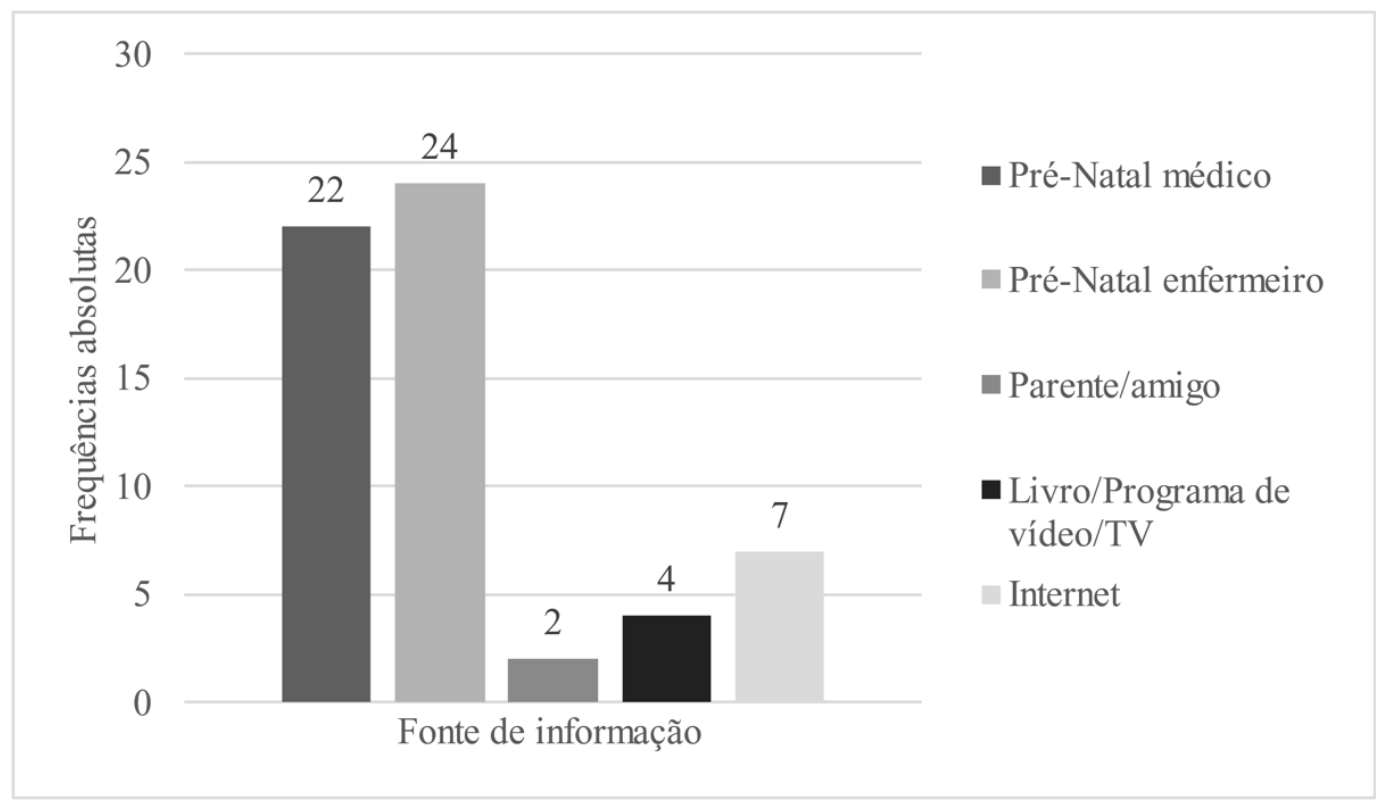

Fonte: Autores.

Com o objetivo de investigar se existia associação entre variáveis do perfil sociodemográfico das entrevistadas, o tipo de parto e o conhecimento sobre analgesia foram realizados testes qui-quadrado $(\chi 2)$. Os resultados demonstraram existir associação estatisticamente significativa entre escolaridade $\left[\chi^{2}(4)=21,45, p<0,001\right]$, renda $\left[\chi^{2}(2)=15,40, p<0,001\right] \mathrm{e}$ trabalho remunerado $[\chi 2(1)=12,37, \mathrm{p}<0,001]$ com o tipo de parto realizado (Tabela 1$)$. As frequências observadas indicaram existir predominância de parto vaginal nas mulheres com ensino médio completo $(87,8 \%)$, renda de dois a três saláriosmínimos $(95,7 \%)$ e que tinham ocupação remunerada $(93,8 \%)$. Não se observou associação do tipo de parto com faixa etária, cor da pele e estado civil $(p=\mathrm{NS})$. Também não foi encontrada associação estatística entre as variáveis do perfil sociodemográfico e a pré-existência de conhecimento (sim; não; não sabia) sobre disponibilidade de analgesia farmacológica durante o parto vaginal, como demonstra a Tabela 2. 
Tabela 1. Perfil sociodemográfico e tipo de parto de puérperas atendidas na maternidade do Hospital Universitário Ana Bezerra, Santa Cruz-RN entre agosto de 2020 e fevereiro de 2021 (n=99).

\begin{tabular}{|c|c|c|c|c|c|c|c|}
\hline \multirow[t]{2}{*}{ Variável } & \multirow[t]{2}{*}{ Categorias } & \multicolumn{2}{|c|}{ Parto Normal } & \multicolumn{2}{|c|}{ Parto Cesariano } & \multirow[t]{2}{*}{$\chi^{2}$} & \multirow[t]{2}{*}{$P$} \\
\hline & & $f$ & $\%$ & $f$ & $\%$ & & \\
\hline \multirow{3}{*}{ Faixa etária } & Adolescentes & 25 & 83,3 & 5 & 16,7 & \multirow{3}{*}{1,44} & \multirow[t]{3}{*}{0,48} \\
\hline & Adultas Jovens & 50 & 78,1 & 14 & 21,9 & & \\
\hline & Adultas & 3 & 60 & 2 & 40 & & \\
\hline \multirow[t]{4}{*}{ Cor da pele } & Branca & 21 & 77,8 & 6 & 22,2 & \multirow[t]{4}{*}{4,17} & \multirow[t]{4}{*}{0,24} \\
\hline & Negra & 2 & 66,7 & 1 & 33,3 & & \\
\hline & Parda & 55 & 80,9 & 13 & 19,1 & & \\
\hline & Indígena & 0 & 0 & 1 & 100 & & \\
\hline \multirow[t]{2}{*}{ Estado Civil } & Solteira & 8 & 66,7 & 4 & 33,3 & \multirow[t]{2}{*}{1,20} & \multirow[t]{2}{*}{0,27} \\
\hline & Casada & 70 & 80,5 & 17 & 19,5 & & \\
\hline \multirow[t]{5}{*}{ Escolaridade } & Ensino fundamental & 14 & 51,9 & 13 & 48,1 & \multirow[t]{5}{*}{21,5} & \multirow[t]{5}{*}{$0,001^{*}$} \\
\hline & Ensino médio incompleto & 20 & 95,2 & 1 & 4,8 & & \\
\hline & Ensino médio completo & 43 & 87,8 & 6 & 12,2 & & \\
\hline & Ensino superior incompleto & 0 & 0 & 1 & 100 & & \\
\hline & Ensino superior completo & 1 & 100 & 0 & 0 & & \\
\hline \multirow[t]{3}{*}{ Renda } & Até 1 salário-mínimo & 33 & 63,5 & 19 & 36,5 & \multirow[t]{3}{*}{15,4} & \multirow[t]{3}{*}{$0,001^{*}$} \\
\hline & 2 a 3 salários-mínimos & 44 & 95,7 & 3 & 4,3 & & \\
\hline & 4 a 6 salários-mínimos & 1 & 100 & 0 & 0 & & \\
\hline \multirow{2}{*}{$\begin{array}{l}\text { Ocupação } \\
\text { remunerada }\end{array}$} & Sim & 45 & 93,8 & 3 & 6,3 & \multirow[t]{2}{*}{12,4} & \multirow[t]{2}{*}{$0,001^{*}$} \\
\hline & Não & 31 & 64,6 & 17 & 35,4 & & \\
\hline
\end{tabular}

*Estatisticamente significante a 1\%; $p$ : nível de significância estatística; $\chi 2$ : Teste qui-quadrado. Fonte: Dados primários da pesquisa (2021) 
Tabela 2. Perfil sociodemográfico e conhecimento sobre alívio farmacológico da dor do trabalho de parto de puérperas atendidas na maternidade do Hospital Universitário Ana Bezerra, Santa Cruz-RN, entre agosto de 2020 e fevereiro de 2021 $(n=99)$.

\begin{tabular}{|c|c|c|c|c|c|c|c|c|c|}
\hline \multirow{3}{*}{ Variáveis } & \multirow{3}{*}{ Categorias } & \multicolumn{6}{|c|}{$\begin{array}{l}\text { Conhecimento sobre analgesia } \\
\text { farmacológica }\end{array}$} & \multicolumn{2}{|c|}{$\begin{array}{l}\text { Estatística } \\
\text { Inferencial }\end{array}$} \\
\hline & & \multicolumn{2}{|c|}{ Sim } & \multicolumn{2}{|c|}{ Não } & \multicolumn{2}{|c|}{ Não sabia* } & \multirow[b]{2}{*}{$\chi^{2}$} & \multirow[b]{2}{*}{$p$} \\
\hline & & $f$ & $\%$ & $f$ & $\%$ & $f$ & $\%$ & & \\
\hline \multirow[t]{4}{*}{ Faixa etária } & Adolescentes & 4 & 13,3 & 18 & 60 & 8 & 26,7 & 2,75 & 0,60 \\
\hline & Adultas Jovens & 10 & 15,6 & 41 & & 13 & 20,3 & & \\
\hline & Adultas & 2 & 40 & 64,1 & & 1 & 20 & & \\
\hline & & & & 2 & 40 & & & & \\
\hline \multirow[t]{8}{*}{ Cor da pele } & Branca & 3 & 11,1 & 18 & & 6 & & 2,27 & 0,89 \\
\hline & Negra & 0 & 0 & 66,7 & & 22,2 & & & \\
\hline & Parda & 13 & 19,1 & 2 & & 1 & & & \\
\hline & Indígena & 0 & 0 & 66,7 & & 33,3 & & & \\
\hline & & & & 40 & & 15 & & & \\
\hline & & & & 58,8 & & 22,1 & & & \\
\hline & & & & 1 & & 0 & & & \\
\hline & & & & 100 & & 0 & & & \\
\hline \multirow[t]{4}{*}{ Estado Civil } & Solteira & 3 & 25 & 8 & & 1 & & 1,90 & 0,38 \\
\hline & Casada & 13 & 14,9 & 66,7 & & 8,3 & & & \\
\hline & & & & 53 & & 21 & & & \\
\hline & & & & 60,9 & & 19,3 & & & \\
\hline \multirow[t]{10}{*}{ Escolaridade } & Fundamental & 4 & 14,8 & 15 & & 8 & & 5,40 & 0,71 \\
\hline & Médio incompleto & 3 & 14,3 & 55,6 & & 29,6 & & & \\
\hline & Médio completo & 9 & 18,4 & 11 & & 7 & & & \\
\hline & Superior & 0 & 0 & 52,4 & & 33,3 & & & \\
\hline & incompleto & 0 & 0 & 33 & & 7 & & & \\
\hline & Superior completo & & & 67,3 & & 14,3 & & & \\
\hline & & & & 1 & & 0 & 0 & & \\
\hline & & & & 100 & & 0 & 0 & & \\
\hline & & & & 1 & & & & & \\
\hline & & & & 100 & & & & & \\
\hline \multirow[t]{6}{*}{ Renda } & Até 1 & 5 & 9,6 & 33 & & 14 & & 4,80 & 0,30 \\
\hline & mínimo & 11 & 23,9 & 63,5 & & 26,9 & & & \\
\hline & 2 a 3 salários- & 0 & 0 & 27 & & 8 & & & \\
\hline & mínimos & & & 58,7 & & 17,4 & & & \\
\hline & 4 a 6 salários- & & & 1 & & 0 & & & \\
\hline & mínimos & & & 100 & & 0 & & & \\
\hline \multirow{4}{*}{$\begin{array}{l}\text { Ucupaçao } \\
\text { remunerada }\end{array}$} & Sim & 8 & 16,7 & 29 & & 11 & & 0,08 & 0,95 \\
\hline & Não & 7 & 14,6 & 60,4 & & 22,9 & & & \\
\hline & & & & 30 & & 11 & & & \\
\hline & & & & 62,5 & & 22,9 & & & \\
\hline
\end{tabular}

*Não sabia responder sim ou não; $p$ : nível de significância estatística; $\chi 2$ : Teste qui-quadrado. Fonte: Dados primários da pesquisa (2021).

Observou-se associação estatisticamente significativa entre a decisão da escolha do parto (vaginal ou cesariano) e o tipo de parto que se realizou efetivamente $[\chi 2(2)=39,97, p<0,001]$. A decisão da gestante sobre a escolha do parto foi mais frequentemente associada com a ocorrência do parto vaginal (100\%), assim como quando a decisão pelo parto vaginal foi compartilhada entre gestante e médico $(89,2 \%)$, como mostra a Tabela 3. 
Tabela 3 - Relação entre o (s) agente (s) da decisão quanto ao tipo de parto e a ocorrência de parto vaginal ou cesariano de puérperas atendidas na maternidade do Hospital Universitário Ana Bezerra, Santa Cruz-RN, entre agosto de 2020 e fevereiro de 2021 ( $\mathrm{n}=99)$.

Tipos de Parto

Estatística inferencial

\begin{tabular}{|c|c|c|c|c|c|c|}
\hline \multirow[t]{2}{*}{$\begin{array}{c}\text { Agente da } \\
\text { decisão quanto } \\
\text { ao tipo de parto }\end{array}$} & \multicolumn{3}{|c|}{ Estatística descritiva - Frequências } & & \multirow[t]{2}{*}{$\chi^{2}$} & \multirow[t]{2}{*}{$P$} \\
\hline & $f$ & $\%$ & $f$ & $\%$ & & \\
\hline Decisão da Gestante & 35 & 100 & 0 & 37 & 39,9 & $0,001 *$ \\
\hline Decisão do Médico & 10 & 37,0 & 17 & 63,0 & & \\
\hline $\begin{array}{l}\text { Decisão } \\
\text { Compartilhada }\end{array}$ & 33 & 89,2 & 4 & 10,8 & & \\
\hline
\end{tabular}

$p$ :estatisticamente significativo.

Fonte: Dados primários da pesquisa (2021).

Demonstrou-se, entretanto, não existir associação entre as variáveis conhecimento da existência de analgesia farmacológica do parto vaginal na maternidade e o tipo de parto realizado $\left[\chi^{2}(2)=1,00, p=0,60\right]$. Do mesmo modo, não se encontrou associação entre pré-existência de conhecimento materno sobre disponibilidade desse tipo de analgesia do processo parturitivo vaginal e sua decisão em relação ao tipo de parto $\left[\chi^{2}(2)=2,09, p=0,39\right]$.

\section{Discussão}

A atenção de qualidade à mulher em processo parturitivo é um direito fundamental e representa um passo indispensável para garantir que ela possa exercer a maternidade com segurança e bem-estar. A dor e a ansiedade desencorajam muitas gestantes a escolher o parto vaginal pois o medo com relação a complicações e o desejo materno pelo parto normal são fatores importantes para reduzir as taxas de cesarianas eletivas (Mascarenhas et al., 2019). Neste escopo temático, o objetivo do presente estudo foi avaliar a existência de conhecimento (intuitivo, elementar) de puérperas em relação à analgesia medicamentosa do parto vaginal, assim como a associação entre esta noção por parte delas e sua decisão quanto ao tipo de parto realizado em uma maternidade de referência no interior do estado do Rio Grande do Norte, Nordeste do Brasil.

A frequência encontrada em relação à existência de conhecimento sobre a disponibilidade da analgesia para o parto foi baixa, mas quantitativamente superior à frequência referente a esta mesma variável em estudos semelhantes realizados em países de economia em desenvolvimento, como Etiópia, Uganda e Índia, que apresentaram frequências de 7 a 9,5\% (Workie et al., 2021; Nabukenya et al., 2015; Prakash et al., 2017; Naithani et al., 2011). Em outro estudo semelhante, também na Índia, mostrou-se uma frequência mais aproximada à encontrada no presente estudo, mesmo que pequena ainda, de $30 \%$ de gestantes em atendimento pré-natal que estavam cientes da existência de analgesia farmacológica para o alívio da dor do parto vaginal (Ponnusamy et al., 2018). Em estudo realizado na Arábia Saudita (Alahmari et al., 2020), mesmo com perfil diferente do da nossa amostra em relação à idade (a maioria das mulheres entre 30 e 40 anos), escolaridade (62,2\% com ensino superior), antecedentes de paridade (88,4\% multíparas), cenário (atenção primária) e sobretudo de diferente cultura, a maioria das gestantes $(62,5 \%)$ não possuía conhecimento sobre a disponibilidade de analgesia obstétrica farmacológica, havendo, contudo, semelhantemente aos resultados do presente estudo, relação significativa entre a noção sobre esta variável e seu nível de escolaridade. 
A tomada de decisão sobre o tipo de parto pela gestante/parturiente depende de diversos fatores, mas o aspecto informacional tem um papel proeminente. Neste sentido, no Brasil, referiu-se que no momento do trabalho de parto, as puérperas recebem orientações gerais de médicos (incluindo obstetras, ginecologistas e pediatras), de enfermeiros e estagiários, mas essas informações não incluem o fator analgesia farmacológica (Pinheiro \& Bittar, 2012). Ao analisar os dados do prénatal no âmbito da Atenção Primária à Saúde no Estado de Santa Catarina, observou-se que as orientações recebidas com maior frequência foram relacionadas aos sinais de risco da gravidez, riscos de automedicação e do tabagismo (Marques et al., 2021), enquanto na sala de pré-parto tem sido, principalmente, sobre a amamentação, cuidados com o bebê, técnicas de respiração e exames realizados (Pinheiro \& Bittar, 2012). A maioria dos adeptos da humanização do parto normal não apreciam positivamente a analgesia farmacológica por considerá-la um processo intervencionista, quando a política nacional vigente busca recuperar o protagonismo da mulher, e não do médico. Independentemente desta questão, é necessário destacar que, a prática da analgesia farmacológica, embora assegurada legalmente, ainda está longe de ser realidade em muitos hospitais, como foi percebido nesta pesquisa.

O emprego de analgesia da dor do trabalho de parto vaginal é escasso em países em desenvolvimento. Estudo empreendido pela Organização Mundial da Saúde, incluindo 357 unidades de saúde em 29 países da África, Ásia, América Latina e Oriente Médio, em 2010 e 2011, mostrou que de quase 221 mil mulheres que tiveram partos vaginais, apenas 4\% foram submetidas à analgesia, sendo a maioria destas originária de países com Índices de Desenvolvimento Humano relativamente mais elevados (Souza, 2018). A prática de manejo da dor do parto é considerada adequada em países desenvolvidos, onde a analgesia farmacológica é considerada uma parte importante dos cuidados pré-natais e intra/pós-parto (Endalew et al., 2020), porém é uma atenção deficiente em países em desenvolvimento, sobretudo os da África Subsaariana (Karn et al., 2016). O fato de as gestantes de países em desenvolvimento desconhecerem a disponibilidade desse modo de alívio farmacológico da dor contribui para a baixa demanda por esse tipo de analgesia. Portanto, em países pobres, o escasso acesso à informação sobre a disponibilidade de determinados cuidados médicos pode influenciar as atitudes em relação ao alívio da dor do parto (Workie et al., 2021). Em um país desenvolvido (Canadá), estudo qualitativo envolvendo parturientes de maternidade de uma comunidade rural mostrou que as gestantes desejavam aprender mais sobre opções de alívio da dor do parto vaginal para fazer uma escolha informada. A maioria das participantes do mencionado estudo expressou o desejo de um parto sem medicamentos, mas enfatizou que tinha "a mente aberta" para considerar a opção de analgesia farmacológica (Munro et al., 2018).

A solicitação materna por analgesia de parto é uma indicação suficiente para sua realização, independentemente da fase do parto e do grau de dilatação da cérvice uterina, conforme as diretrizes nacionais de assistência ao parto normal (Brasil, 2017), o que inclui parturientes em fase latente com dor intensa, após esgotados os métodos não farmacológicos. A aparente "liberdade de escolha" outorgada à mulher nos programas de humanização hospitalar muitas vezes é caracterizada pela falta de informações adequadas e, portanto, não poderia configurar o que se denomina escolha propriamente informada ou consentimento esclarecido (Mandarino et al., 2009). O Ministério da Saúde do Brasil recomenda que as mulheres devem saber que existem formas para se controlar a dor associada ao trabalho de parto normal e que não há justificativa para se realizar uma cesariana apenas com esta finalidade, e, portanto, esses métodos para alívio da dor devem estar disponíveis para essas mulheres se houver necessidade (Brasil, 2001b).

Por outro lado, a percepção da dor do parto e seu alívio é diferente entre mulheres culturalmente diversas. Embora não esteja bem documentado, existe uma noção entre as várias divisões culturais de que as mulheres devem estar preparadas para suportar estoicamente a dor do parto, ainda que seja considerada muito forte, ou insuportável, por quase todas elas (Yadav et al., 2018). No nosso estudo, não se observou uma lacuna expectativa-experiência em relação à dor, a partir da pontuação em uma escala unidimensional em que a dor autorrelatada apresentou escores elevados, mesmo que as participantes tenham 
revelado que a intensidade da dor experienciada correspondeu às suas expectativas, levando-se em conta que geralmente a experiência costuma ser muito diferente da expectativa em se tratando de dor (Lally et al., 2008). Trata-se de uma boa escala para medir mudanças da intensidade da dor, não sendo, contudo, ideal para uma única medida em um evento dinâmico como o parto, em que a dor aumenta progressivamente, e referida de modo retrospectivo, após o parto, a mulher tende a se remeter ao momento de maior dor quando questionada, o que pode ter ocorrido no presente estudo.

Em revisão sistemática das expectativas e experiências das mulheres em relação à dor e seu alívio no trabalho de parto, Lally et al. (2008) destacaram a discrepância entre expectativas e experiências de dor: entre as mulheres que planejaram não usar analgesia farmacológica, 52\% o fizeram. Os referidos autores concluíram que o conhecimento sobre o manejo da dor ajudou as mulheres a fazerem escolhas informadas e que a consideração e avaliação das opções também as ajudaram a lidar física e psicologicamente com a dor do parto. A mencionada revisão contém análise de estudos mostrando que as expectativas das mulheres em relação ao alívio da dor se associaram ao desejo de acesso a um alívio analgésico eficaz, similarmente aos resultados observados no nosso estudo. No estudo intitulado "Nascer no Brasil" (Leal \& Gama, 2014) mostrou que o principal motivo para a preferência das mulheres pela cesariana é o medo da dor do parto e que o modelo de atenção vigente apresenta baixa utilização de tecnologias que favorecem a progressão fisiológica do trabalho de parto. Portanto, segundo o mesmo estudo "uma das premissas para redução das cesarianas no Brasil é a melhoria da assistência ao trabalho de parto e ao parto vaginal visando à promoção da autonomia, do protagonismo e do bem-estar das mulheres.” Apesar de a adoção de algumas práticas recomendadas pela Organização Mundial da Saúde terem surtido efeito, a que representou menor crescimento foi a oferta de analgesia peridural (apenas $16 \%$ no setor público), sendo sugerida a sua implementação como aliada no incentivo ao parto vaginal.

Métodos complementares de alívio da dor do parto também são largamente difundidos em todo o mundo, em uma variedade de técnicas não-farmacológicas. Em estudo de abordagem qualitativa, Almeida et al. (2015) enfocaram o conhecimento de puérperas sobre tais métodos em uma maternidade do interior do estado de São Paulo, onde elas foram estimuladas para a prática dessas medidas, com primazia do papel assistencial do profissional de enfermagem e obstetrizes nessas ações, o que se assemelha, de certo modo, ao resultado observado no nosso estudo, em que houve maior protagonismo do enfermeiro no que concerne à ação educativa em saúde para as gestantes. Gestantes atendidas em consultas conjuntas por profissionais médico e enfermeiro apresentaram chance $41 \%$ superior de adequação às orientações, em comparação com aquelas atendidas exclusivamente por médicos (Marques et al., 2021). Da mesma forma, Marins et al.(2020) avaliaram o conhecimento de puérperas a respeito dos métodos de alívio da dor na parturição e concluíram que, apesar de receberem orientações no pré-natal em relação ao parto, ainda carecem de entendimento e compreensão de questões relativas aos tipos de tecnologias de alívio da dor e sua aplicabilidade e, por isso, os citados autores defendem a oferta de mais informações e orientações mais acessíveis acerca dessas tecnologias de cuidado e alívio da dor do parto pelos profissionais de saúde na fase do pré-natal.

Contudo, reconhece-se o direito da mulher também à abordagem farmacológica, quando as medidas não farmacológicas forem insuficientes para confortá-la no trabalho de parto (Felisbino-Mendes et al., 2017). A garantia legal, independentemente do que se propaga a favor ou contra a utilização da analgesia, este recurso previsto em lei não está sendo praticado, uma vez que sequer é possível o exercício pelas mulheres de decidirem se desejam ou não alívio farmacológico, considerando que não é fornecido este tipo de orientação de seus direitos (Marques et al., 2021). As principais justificativas para a indisponibilidade da analgesia farmacológica de parto têm sido a falta de insumos, estrutura de suporte e monitorização adequados, treinamento da equipe e profissionais especializados (Rocha \& Ferreira, 2020), porém existem recursos humanos habilitados na maternidade do HUAB para realização deste tipo de assistência, ainda que não existam protocolos clínicos locais e todos os insumos para que se torne rotineira a sua oferta. 
Para compreender o cenário de assistência ao parto, é importante identificar os perfis sociodemográficos dos atores envolvidos, em que contexto se inserem e quais são as características predominantes de cada local e serviço (Rocha \& Ferreira, 2020). Em algumas culturas, a dor associada ao parto é considerada natural e, assim, a ideia de aboli-la com medicamentos parece desnecessária ou contrária a determinados valores tradicionais locais. Portanto, a forma mais comum de alívio da dor em nações em desenvolvimento é o apoio contínuo de acompanhante durante o trabalho de parto (Brüggemann et al., 2005). Nesse sentido, em estudo qualitativo, Pinheiro e Bittar (2012) mostraram que a experiência da parturição foi percebida pelas mulheres como extremamente dolorosa e sofrida, porém compensada, no entanto, pela atenção e apoio recebidos de profissionais e acompanhantes, que contribuíram para uma visão satisfatória do parto normal sem analgesia.

O perfil sociodemográfico das mulheres avaliadas nos estudos mencionados anteriormente, com o objetivo de comparação das frequências em que há informação sobre analgesia farmacológica, é análogo ao que foi observado no presente trabalho: jovens, casadas, com nível médio de instrução, sem ocupação remunerada e baixa renda. De acordo com o padrão de prática de cuidados obstétricos nos países onde foram realizados os referidos estudos (Workie et al., 2021; Nabukenya et al., 2015; Prakash et al., 2017; Naithani et al., 2011), o fornecimento de suporte físico e psicológico e o uso de medidas farmacológicas e não farmacológicas durante o trabalho de parto vaginal são tidos como competências críticas essenciais do cuidado, porém sua prática real é considerada insatisfatória (Kayiga et al., 2016; Austin et al., 2015; Nair \& Panda, 2011). O perfil sociodemográfico da nossa amostra também é semelhante ao de pesquisas realizadas no Brasil, em maternidades do interior do estado de São Paulo, com o objetivo de avaliar o conhecimento de puérperas com relação aos métodos não farmacológicos de alívio da dor do parto (Almeida et al., 2015), assim como em maternidades públicas de Maceió, Alagoas, Nordeste do Brasil (Kassar et al., 2006), em unidades básicas de saúde do interior do Rio Grande do Sul (Weidle et al., 2014) e de Brasília-DF (Tostes \& Seidl, 2016) e maternidade pública de São Luiz, Maranhão (Mandarino et al., 2009). Trata-se de mulheres com um perfil considerado de menor risco obstétrico, por ser esta faixa etária a melhor época para procriação do ponto de vista biológico e social (Kassar et al., 2006).

Ainda na análise da associação entre variáveis do perfil sociodemográfico com o tipo de parto ou a escolha da modalidade de parto, a informação sobre cor/raça foi coletada devido às disparidades previamente documentadas em relação a esta variável social, ainda que não haja implicações biológicas envolvidas nas diferenças. Mulheres que se autodeclararam de cor/raça negra ou parda, mesmo quando feito controle pela educação formal e outras variáveis, tiveram iniquidades no processo de atenção à gestação e ao parto, evidenciando um gradiente em comparação com mulheres brancas (Leal et al., 2017; Diniz et al., 2016). Ainda que não tenha sido observada diferença estatística entre as categorias consideradas no nosso estudo em relação a esta variável, detectando-se diferenças quanto à escolaridade, renda e trabalho remunerado, pode não ter havido dados suficientes para explicar uma diferença quanto à cor/raça, pois havia poucas participantes que se autodeclararam negras e tinha apenas uma indígena na amostra. Similarmente, pode ter ocorrido o mesmo em relação à falta de detecção de diferenças no que concerne à variável faixa de idade, pois havia uma minoria da amostra na categoria adulta (acima de 40 anos). Assim, propõe-se a realização de futuros estudos que enfoquem tais variáveis sociodemográficas em amostras maiores e com maior diversidade nos referidos constructos.

A maioria das puérperas do nosso estudo preferiu a via de parto vaginal, achado que coincide com as respostas de gestantes de quatro unidades básicas de saúde de um município do interior da região Sul do Brasil (Weidle et al., 2014) e de Brasília-DF (Tostes \& Seidl, 2016). A observação da predominância de parto vaginal nas mulheres com ensino médio completo, que tinham renda acima de um salário-mínimo e ocupação remunerada, é compatível com achados de Weidle et al. (2014) em maternidade do Rio Grande do Sul e de Mandarino et al. (2009) no Maranhão. Contudo, a preferência referida pelas gestantes deste último estudo mencionado não se associou com o tipo de parto realizado, com ocorrência de quase $90 \%$ de partos cesarianos na amostra de 81 mulheres, contrariamente ao que foi encontrado na nossa pesquisa. É relevante destacar 
que, como afirmam Faisal-Cury e Menezes (2006), "a escolha do tipo de parto pela gestante é assunto atual, complexo e polêmico" e, portanto, o caráter discriminante das condições socioeconômicas pode ser aparentemente compreendido quando os resultados da assistência ao parto vaginal são relacionados aos estratos de renda e à escolaridade das mães, mesmo dentro do mesmo serviço público de saúde (Caminha et al., 2008). Por outro lado, a tomada de decisão da escolha do tipo de parto geralmente se dá em dois momentos; primeiro, durante o período pré-natal, quando as gestantes formam uma preferência; e então durante o trabalho de parto, onde fazem uma escolha real. Devido à incerteza do trabalho de parto e do parto, as mulheres geralmente sentem que suas necessidades muitas vezes não podem ser previstas com antecedência (Munro et al., 2018) e tendem a mudar ao longo da gestação, havendo associação com os vínculos sociais da gestante (Domingues et al., 2014).

A maioria das participantes da amostra não era primípara e teve assistência pré-natal com número de consultas adequado, conforme recomendação do Ministério da Saúde (Brasil, 2005), além de ter apresentado parto vaginal, conforme seu desejo. Outros estudos brasileiros, não obstante, encontraram prevalências elevadas de resultados insatisfatórios na adequação do pré-natal (Dias-da-Costa et al., 2013; Chrestani et al., 2008; Coimbra et al., 2003). As frequências referentes a estas variáveis obstétricas encontradas no nosso estudo também foram melhores que as demonstradas em estudo realizado nas regiões Norte e Nordeste do país, onde se reportou que 48,2\% das gestantes fizeram menos de seis consultas no pré-natal (Chrestani et al., 2008). Esse aspecto é fundamental, tendo em vista a importância da assistência pré-natal no sentido de representar uma oportunidade privilegiada de informar às mulheres e prepará-las para o parto e puerpério, incluindo fornecimento de informações acerca das vantagens e desvantagens de cada tipo de parto, bem como sobre os procedimentos recomendados durante a assistência ao parto e nascimento. Apesar disso, informações obtidas pelas mulheres nos serviços de saúde nem sempre contribuem para uma escolha informada, de modo que elas possam conhecer todos os benefícios e prejuízos dos procedimentos que eventualmente serão realizados na maternidade hospitalar (Vidal, 2017). Sob esta perspectiva, um processo educativo de qualidade deveria fazer parte da atenção primária à saúde e do cuidado pré-natal e de trabalho de parto em maternidades.

Na maior parte de nossa amostra, parece ter havido escasso fornecimento de informações às mulheres sobre analgesia farmacológica, mas em relação ao contingente da amostra que referiu ter sido informada, isto se deu sobretudo no pré-natal e com protagonismo por parte do enfermeiro da equipe. Similarmente, entre aquelas que manifestaram desejo de receber alívio farmacológico da dor durante o parto, somente cerca de um terço recebeu orientação sobre o assunto no pré-natal. A equipe de saúde, portanto, deve estar diretamente implicada neste processo de informar e prestar esclarecimentos acerca do ciclo gravídico-puerperal, considerando também a disponibilidade de analgesia farmacológica para as mulheres que, estando informadas, podem ou não decidir obtê-la. Uma maioria significativa de puérperas incluídas em outro estudo disse que gostaria de ter analgesia no parto, porém a maioria (47\%) das que sabiam sobre o assunto obteve a informação de amigos e familiares (Nabukenya et al., 2015), diferindo dos nossos resultados. Alshahrani (2019) defende a importância de educar as gestantes e transmitir informações sobre as técnicas de alívio da dor no parto visando diminuir a incidência de cesariana a pedido.

Por outro lado, a frequência de partos vaginais observada no presente estudo também foi superior ao reportado na literatura brasileira. A realização de cesarianas sem indicação obstétrica é comum no Brasil, sendo este o tipo de parto predominante no país desde 2009, considerando serviços públicos e privados de saúde (Carvalho et al., 2014). De todos os partos realizados no Sistema Único de Saúde entre 2000 e 2018, 51,3\% foram partos vaginais (Guimarães et al., 2021), mas em estudo epidemiológico envolvendo dados oficiais dos partos ocorridos entre 2011 e 2015 (99,9\% hospitalares) em 25 municípios do Rio Grande do Norte (Seridó potiguar, mesorregião vizinha a da localização do HUAB), observou-se que 58\% foram cesarianos (Ribeiro et al., 2019). O cenário de assistência obstétrica no âmbito de maternidades públicas do estado do Rio Grande do Norte é considerado predominantemente intervencionista (Carvalho \& Silva, 2019). Entretanto, é preciso 
destacar que o local onde se realizou o nosso estudo, o Hospital Universitário Ana Bezerra (HUAB), é um uma instituição de referência regional na atenção à saúde materno-infantil e campo de formação de profissionais de saúde, além de se basear na Política Nacional de Humanização (PNH) e de ter implantado o Projeto de Gestão Humanizada desde 1999. Esta instituição é referência hospitalar em atendimento secundário à Gestante de Alto Risco, assim como é habilitada como unidade que realiza assistência ao parto sem distócia (Brasil, 2013). Além disso, a maternidade do HUAB recebeu prêmios importantes, como o outorgado pelo Fundo das Nações Unidas para a Infância (UNICEF) como "Hospital Amigo da Criança" em 1996 e o Prêmio Nacional Galba de Araújo, conferido pelo Ministério da Saúde a instituições que valorizam o parto humanizado de maneira natural, como potencial exemplo para outros grandes hospitais brasileiros (Brasil, 2001). As diretrizes da PNH (Brasil, 2008) têm por princípio o fortalecimento e protagonismo dos usuários, o que é essencial para a promoção da cultura da humanização no cuidado em saúde.

Se por um lado, se trata de uma amostra com possível viés de seleção, por se tratar de usuárias de um serviço de referência regional e nacional em termos de políticas de humanização hospitalar, por outro, evidencia-se de forma ainda mais incisiva que a frequência de apenas $22,2 \%$ de puérperas que sabiam da existência de analgesia farmacológica do parto vaginal é precária, uma vez que a comunicação é parte essencial do cuidado humanizado em saúde (Deslandes \& Mitre, 2009) e considerando que todas as participantes da nossa amostra realizaram acompanhamento pré-natal. As mudanças nos modos de gestão e atenção obstétricas nas maternidades decorrem de um aprimoramento dos mecanismos de diálogo e qualificação nas comunicações interpessoais entre profissionais da saúde e usuárias, o que inclui orientar sobre os diferentes métodos de alívio da dor, respeitando a escolha da mulher para o uso, ou não, do suporte farmacológico disponível na maternidade sob assistência de anestesistas (Trindade et al., 2018). A analgesia farmacológica do parto difundiu-se em muitos centros obstétricos brasileiros, alcançando taxas acima de 50\%, particularmente mais elevada em primigestas, e havendo, paralelamente, diminuição das taxas de cesarianas eletivas, o que provavelmente reflete aspectos psicoemocionais das parturientes em relação ao enfrentamento da dor (Leão, 2016).

São necessários novos estudos, de preferência incluindo a oferta de alívio farmacológico da dor na atenção às parturientes do nosso serviço, bem como a educação das gestantes em relação aos métodos de alívio da dor do parto pelos profissionais da assistência pré-natal. Essa ação de educação em saúde pode ser feita também por meio da distribuição de folhetos informativos nas unidades básicas de saúde e maternidades onde se realizam as consultas pré-natais, pelos obstetras, enfermeiros, anestesiologistas e parteiras. A preocupação atual com a humanização no parto implica dar liberdade às escolhas da parturiente, prestar um atendimento focado em suas necessidades, aliviar seus anseios, esclarecer as suas dúvidas.

É possível contribuir para o aprimoramento dos serviços de assistência pré-natal de forma a aproximá-los das necessidades integrais das gestantes e das recomendações do Programa de Humanização do Pré-natal e Nascimento. Políticas públicas simples e bem conduzidas podem mudar o cenário da atenção ao parto e ao nascimento, favorecer um aumento no nível de conhecimento das pacientes e prepará-las melhor para os desafios, garantir maior qualidade e bem-estar à parturiente, bem como ajudar a reduzir o índice de cesarianas desnecessárias. Durante o pré-natal, é preciso informar as mulheres sobre riscos e benefícios das diversas práticas e intervenções durante o trabalho de parto e parto, a necessidade de escolha de um acompanhante pela mulher para o apoio durante o parto, estratégias de controle da dor e métodos disponíveis na maternidade, descrevendo os riscos e benefícios de cada método (farmacológicos e não farmacológicos), organização e indicadores assistenciais do local de atenção ao parto, limitações (física, recursos disponíveis) relativos à unidade, bem como da disponibilidade de certos métodos e técnicas para auxiliar as mulheres em escolhas bem informadas (Brasil, 2017).

Os resultados desta pesquisa, que abrangeu apenas um serviço hospitalar público, ainda que este seja o único existente na cidade de Santa Cruz-RN, não podem ser extrapolados para toda a população; no entanto, os achados demonstrados 
conferem importância ao tema e apontam claramente para a necessidade da realização de estudos de maior porte, que permitam um conhecimento mais amplo da nossa realidade quanto ao problema de pesquisa proposto.

\section{Conclusão}

A frequência de informação a respeito da existência de alívio farmacológico da dor do parto é baixa por parte de parturientes atendidas na maternidade do HUAB, uma instituição de referência regional na atenção à saúde materno-infantil. $\mathrm{O}$ conhecimento materno sobre a disponibilidade de alívio da dor durante o trabalho de parto se relacionou com nível de escolaridade, renda e trabalho remunerado, mas não se associou com a decisão materna pelo parto vaginal ou com a realização deste tipo de parturição. Portanto, com em relação à principal questão de pesquisa, constatou-se que o conhecimento materno sobre a disponibilidade de alívio medicamentoso da dor durante o trabalho de parto é baixo, porém não foi possível demonstrar que esta variável se relaciona com a ocorrência de parto cesariano.

A educação em saúde sobre os benefícios da analgesia do parto deve ser incentivada na instituição. Considera-se que este estudo contribui para retratar a distribuição da variável primária estudada e uma melhor compreensão dos fatores sociodemográficos associados à preferência pela via do parto vaginal.

São necessários novos estudos, de preferência incluindo a oferta de alívio farmacológico da dor na atenção às parturientes do nosso serviço, bem como a educação das gestantes em relação aos métodos de alívio da dor do parto.

\section{Referências}

Ali Alahmari, S. S., ALmetrek, M., Alzillaee, A. Y., Hassan, W. J., \& Ali Alamry, S. M. (2020). Knowledge, attitude, and practice of childbearing women toward epidural anesthesia during normal vaginal delivery in Alsanayeah Primary Health Care in Khamis Mushait. Journal of Family Medicine and Primary Care, 9(1), 99-104. https://doi.org/10.4103/jfmpc.jfmpc_530_19

Almeida, J. M. de, Acosta, L. G., \& Pinhal, M. G. (2015). Conhecimento das puérperas com relação aos métodos não farmacológicos de alívio da dor do parto. Revista Mineira de Enfermagem, 19(3), 711-724. https://doi.org/10.5935/1415-2762.20150054

Alshahrani, M. S. (2019). An evaluation of the different types of labor pain relief, preferred methods of pain relief, and effects of social media on awareness and knowledge among pregnant women. A cross-sectional study in the Kingdom of Saudi Arabia. Saudi Medical Journal, 40(9), 914-921. https://doi.org/10.15537/smj.2019.9.24511

Austin, A., Gulema, H., Belizan, M., Colaci, D. S., Kendall, T., Tebeka, M., Hailemariam, M., Bekele, D., Tadesse, L., Berhane, Y., \& Langer, A. (2015). Barriers to providing quality emergency obstetric care in Addis Ababa, Ethiopia: Healthcare providers' perspectives on training, referrals and supervision, a mixed methods study. BMC Pregnancy and Childbirth, 15, 74. https://doi.org/10.1186/s12884-015-0493-4

Brasil. (2019). Carta de Serviços ao Cidadão do Hospital Universitário Ana Bezerra (HUAB). Ministério da Educação. https://www.gov.br/ebserh/ptbr/hospitais-universitarios/regiao-nordeste/huab-ufrn/acesso-a-informacao/programas-e-projetos/CartadeServios2020.pdf

Brasil. (2017). Diretrizes Nacionais de Assistência Ao Parto Normal. Ministério da https://bvsms.saude.gov.br/bvs/publicacoes/diretrizes_nacionais_assistencia_parto_normal.pdf

Brasil. (2013). Plano de Reestruturação do Hospital Universitário Ana Bezerra da Universidade Federal do Rio Grande do Norte. Ministério da Educação.http://ebserh.mec.gov.br/images/pdf/contratos_adesao_huf/ufrn/huab/plano_de_reestruturacao_huab_ufrn.pdf

Brasil. (2006). Pré-natal e puerpério: atenção qualificada e humanizada: manual técnico. Ministério da Saúde.https://bvsms.saude.gov.br/bvs/publicacoes/manual_pre_natal_puerperio_3ed.pdf

Brasil. (2005). Pré-natal e Puerpério, Atenção Qualificada e Humanizada. Ministério da Saúde. https://bvsms.saude.gov.br/bvs/publicacoes/prenatal_puerperio_atencao_humanizada.pdf

Brasil. (2001). II Prêmio Galba de Araújo: O reconhecimento para quem valoriza o parto humanizado. Ministério da Saúde.https://bvsms.saude.gov.br/bvs/publicacoes/reconhecimento_quem_valoriza_parto_humanizado.pdf

Brasil. (2001). Parto, Aborto e Puerpério, Assistência Humanizada à Mulher. Ministério da Saúde. https://bvsms.saude.gov.br/bvs/publicacoes/cd04_13.pdf

Brüggemann, O. M., Parpinelli, M. A., \& Osis, M. J. D. (2005). Evidências sobre o suporte durante o trabalho de parto/parto: Uma revisão da literatura. Cadernos de Saúde Pública, 21, 1316-1327. https://doi.org/10.1590/S0102-311X2005000500003

Caminha, M. de F. C., Figueira, M. C. dos S., Santos, L. G. de A. dos, Albuquerque, E. C. de, Lira, P. I., \& Filho, M. B. (2008). Assistência ao parto normal no Estado de Pernambuco: Aspectos geográficos, socioeconômicos e profissionais, com ênfase no papel da enfermeira. Epidemiologia e Serviços de Saúde, 17(3), 195-204. https://doi.org/10.5123/S1679-49742008000300005 
Carvalho, I. da S., Costa Júnior, P. B. da, Macedo, J. B. P. de O., \& Brito, R. S. de. (2014). Caracterização epidemiológica de partos e nascimentos: Estudo ecológico com base em um sistema de informação. Rev. enferm. UFPE on line, 616-623.

Carvalho, J. B. L. \& Silva, F. C. B (2019). Experiência exitosa na coordenação do curso de especialização em Enfermagem Obstétrica: Rede Cegonha III pela UFRN por meio do processo de formação/intervenção In Carvalho, J. B. L. de. \& Silva, F. C. B. da. (Org) (Re)ssignificando a prática da enfermagem obstétrica: Processo de Formação-Intervenção (pp. 15-22). Caule de Papiro.

Chrestani, M. A. D., Santos, I. S., Cesar, J. A., Winckler, L. S., Gonçalves, T. S., \& Neumann, N. A. (2008). Assistência à gestação e ao parto: Resultados de dois estudos transversais em áreas pobres das regiões Norte e Nordeste do Brasil. Cadernos de Saúde Pública, 24, 1609-1618. https://doi.org/10.1590/S0102$311 \mathrm{X} 2008000700016$

Coimbra, L. C., Silva, A. A. M., Mochel, E. G., Alves, M. T. S. S. B., Ribeiro, V. S., Aragão, V. M. F., \& Bettiol, H. (2003). Fatores associados à inadequação do uso da assistência pré-natal. Revista de Saúde Pública, 37, 456-462. https://doi.org/10.1590/S0034-89102003000400010

Costa, R., Figueredo, B., Pacheco, A., Marques, A. \& Pais, A. (2004). Questionário de Experiência e Satisfação com o Parto (Qesp). Psicologia, Saúde \& Doenças, 5 (1): 159-187

Deslandes, S. F., \& Mitre, R. M. de A. (2009). Processo comunicativo e humanização em saúde. Interface - Comunicação, Saúde, Educação, 13, 641-649. https://doi.org/10.1590/S1414-32832009000500015

Dias-da-Costa, J. S., Cesar, J. A., Haag, C. B., Watte, G., Vicenzi, K., \& Schaefer, R. (2013). Inadequação do pré-natal em áreas pobres no Nordeste do Brasil: Prevalência e alguns fatores associados. Revista Brasileira de Saúde Materno Infantil, 13, 101-109. https://doi.org/10.1590/S1519-38292013000200003

Diniz, C. S. G., Batista, L. E., Kalckmann, S., Schlithz, A. O. C., Queiroz, M. R., \& Carvalho, P. C. de A. (2016). Desigualdades sociodemográficas e na assistência à maternidade entre puérperas no Sudeste do Brasil segundo cor da pele: Dados do inquérito nacional Nascer no Brasil (2011-2012)1. Saúde e Sociedade, 25, 561-572. https://doi.org/10.1590/S0104-129020162647

Domingues, R. M. S. M., Dias, M. A. B., Nakamura-Pereira, M., Torres, J. A., d'Orsi, E., Pereira, A. P. E., Schilithz, A. O. C., \& Carmo Leal, M. do. (2014). Process of decision-making regarding the mode of birth in Brazil: From the initial preference of women to the final mode of birth. Cadernos De Saude Publica, 30 Suppl 1, S1-16. https://doi.org/10.1590/0102-311x00105113

Endalew, N. S., Tawuye, H. Y., \& Melesse, D. Y. (2020). Knowledge and attitude towards pain relief in labor among final year midwifery students: A crosssectional study. International Journal of Surgery Open, 24, 38-42. https://doi.org/10.1016/j.ijso.2020.03.006

Faisal-Cury, A., \& Menezes, P. R. (2006). Fatores associados à preferência por cesareana. Revista de Saúde Pública, 40, 226-232. https://doi.org/10.1590/S0034-89102006000200007

Felisbino-Mendes, M. S., Santos, L. O., Amorim, T., Costa, I. N., \& Martins, E. F. (2017). O uso de analgesia farmacológica influencia no desfecho de parto? Acta Paulista de Enfermagem, 30, 458-465. https://doi.org/10.1590/1982-0194201700067

Freitas, J. F. de, \& Meinberg, S. (2009) Analgesia de parto: Bloqueios locorregionais e analgesia sistêmica. Revista Médica de Minas Gerais, 19 (3), 7-14.

Guimarães, N. M., Freitas, V. C. de S., Senzi, C. G. de, Frias, D. F. R., Gil, G. T., \& Lima, L. D. dos S. C. (2021). Partos no sistema único de saúde (SUS) brasileiro: Prevalência e perfil das partutientes / Childbirths under the unified health system (SUS) of brazil: prevalence and profile of parturients. Brazilian Journal of Development, 7(2), 11942-11958. https://doi.org/10.34117/bjdv7n2-019

IBGE - Instituto Brasileiro de Geografia e Estatística. (2016). Santa Cruz. IBGE Cidades. https://cidades.ibge.gov.br/brasil/pb/santa-cruz/panorama.

Karn, S., Yu, H., Karna, S., Chen, L., \& Qiao, D. (2016). Women's Awareness and Attitudes towards Labor Analgesia Influencing Practice between Developed and Developing Countries. Advances in Reproductive Sciences, 4(2), 46-52. https://doi.org/10.4236/arsci.2016.42007

Kassar, S. B., Lima, M. de C., Albuquerque, M. de F. M. de, Barbieri, M. A., \& Gurgel, R. Q. (2006). Comparações das condições socioeconômicas e reprodutivas entre mães adolescentes e adultas jovens em três maternidades públicas de Maceió, Brasil. Revista Brasileira de Saúde Materno Infantil, 6, 397403. https://doi.org/10.1590/S1519-38292006000400006

Kayiga, H., Ajeani, J., Kiondo, P., \& Kaye, D. K. (2016). Improving the quality of obstetric care for women with obstructed labour in the national referral hospital in Uganda: Lessons learnt from criteria based audit. BMC Pregnancy and Childbirth, 16(1), 152. https://doi.org/10.1186/s12884-016-0949-1

Lally, J. E., Murtagh, M. J., Macphail, S., \& Thomson, R. (2008). More in hope than expectation: A systematic review of women's expectations and experience of pain relief in labour. BMC Medicine, 6, 7. https://doi.org/10.1186/1741-7015-6-7

Leal, M. do C., Gama, S. G. N. da, Pereira, A. P. E., Pacheco, V. E., Carmo, C. N. do, \& Santos, R. V. (2017). A cor da dor: Iniquidades raciais na atenção pré-natal e ao parto no Brasil. Cadernos de Saúde Pública, 33. https://doi.org/10.1590/0102-311X00078816

Leal, M. do C., \& Gama, S. G. N. da. (2014). Nascer no Brasil. Cadernos de Saúde Pública, 30, S5-S5. https://doi.org/10.1590/0102-311XED01S114

Leão, B. C. C. (2016). Influência da analgesia de parto no desfecho obstétrico: Estudo em uma maternidade pública brasileira. [Dissertação de Mestrado, Universidade Federal de Minas Gerais. Repositório Institucional da UFMG. https://repositorio.ufmg.br/handle/1843/BUOS-AJNP8R

Mandarino, N. R., Chein, M. B. da C., Monteiro Júnior, F. das C., Brito, L. M. O., Lamy, Z. C., Nina, V. J. da S., Mochel, E. G., \& Figueiredo Neto, J. A. de. (2009). Aspectos relacionados à escolha do tipo de parto: Um estudo comparativo entre uma maternidade pública e outra privada, em São Luís, Maranhão, Brasil. Cadernos de Saúde Pública, 25, 1587-1596. https://doi.org/10.1590/S0102-311X2009000700017

Marques, B. L., Tomasi, Y. T., Saraiva, S. dos S., Boing, A. F., \& Geremia, D. S. (2021). Orientações às gestantes no pré-natal: A importância do cuidado compartilhado na atenção primária em saúde. Esc. Anna Nery Rev. Enferm, e20200098-e20200098. 
Mascarenhas, V. H. A., Lima, T. R., Silva, F. M. D. e, Negreiros, F. dos S., Santos, J. D. M., Moura, M. Á. P., Gouveia, M. T. de O., \& Jorge, H. M. F. (2019) Evidências científicas sobre métodos não farmacológicos para alívio a dor do parto. Acta Paul. Enferm. (Online), 350-357.

Monte, A. S. (2013). Adaptação e validação de conteúdo do "Questionário para as mulheres" para a realidade da assistência à mulher no pré, trans e pósparto em Fortaleza. [Dissertação de Mestrado, Universidade Estadual do Ceará. Repositório Institucional da UECE. http://www.uece.br/cmacclis/dmdocuments/ALANASANTOSMONTE.pdf

Munro, S. B., Hui, A., Gemmell, E. A., Torabi, N., Johnston, A. S., \& Janssen, P. A. (2018). Evaluation of an Information Pamphlet for Women Considering Epidural Analgesia in Labour. Journal of Obstetrics and Gynaecology Canada: JOGC, 40(2), 171-179. https://doi.org/10.1016/j.jogc.2017.06.028

Nabukenya, M. T., Kintu, A., Wabule, A., Muyingo, M. T., \& Kwizera, A. (2015). Knowledge, attitudes and use of labour analgesia among women at a lowincome country antenatal clinic. BMC Anesthesiology, 15, 98. https://doi.org/10.1186/s12871-015-0078-9

Nair, H., \& Panda, R. (2011). Quality of maternal healthcare in India: Has the National Rural Health Mission made a difference? Journal of Global Health $1(1), 79-86$.

Naithani, U., Bharwal, P., Chauhan, S. S., Kumar, D., Gupta, S., \& Kirti. (2011). Knowledge, attitude and acceptance of antenatal women toward labor analgesia and caesarean section in a medical college hospital in India. Journal of Obstetric Anaesthesia and Critical Care, 1(1), 13. https://doi.org/10.4103/2249-4472.84250

Pinheiro, B. C., \& Bittar, C. M. L. (2012). Percepções, expectativas e conhecimentos sobre o parto normal: Relatos de experiência de parturientes e dos profissionais de saúde. Aletheia, 37, 212-227.

Ponnusamy, R., Reddy, H., \& Murugesan, R. (2018). Awareness, knowledge and attitude about labor analgesia among providers and parturients , a survey based study. Anaesthesia, Pain \& Intensive Care. https://www.semanticscholar.org/paper/Awareness\%2C-knowledge-and-attitude-about-labor-amongPonnusamy-Reddy/2f1cf85e893b1d324e395984ea88993dcf8f57ec

Prakash, A., Yadav, A., Karim, H. M. R., Sahoo, S. K., Jena, P., \& Aman, K. (2017). Knowledge, Awareness and Acceptance of Labor Analgesia among Antenatal Women in a Remote Island: A Questionnaire Based Study. British Journal of Medicine \& Medical Research, 21(10), 1-7.

Ribeiro, E. da C., Sousa, A. C. P. de A., Dantas, L. de A., Marcelino, S. A., Lima, L. T., \& Ferreira, G. O. (2019). Análise epidemiológica dos partos em uma região do nordeste brasileiro. Revista Recien - Revista Cientifica de Enfermagem, 9(28), 64-73. https://doi.org/10.24276/rrecien2358-3088.2019.9.28.64-73

Rocha, N. F. F. da, \& Ferreira, J. (2020). A escolha da via de parto e a autonomia das mulheres no Brasil: Uma revisão integrativa. Saúde em Debate, 44, 556568. https://doi.org/10.1590/0103-1104202012521

Shaaban, O. M., Abbas, A. M., Mohamed, R. A., \& Hafiz, H. (2017). Lack of pain relief during labor is blamable for the increase in the women demands towards cesarean delivery: A cross-sectional study. Facts, Views \& Vision in ObGyn, 9(4), 175-180.

Sousa, V. D., Driessnack, M., \& Mendes, I. A. C. (2007). Revisão dos desenhos de pesquisa relevantes para enfermagem: Parte 1: desenhos de pesquisa quantitativa. Revista Latino-Americana de Enfermagem, 15, 502-507. https://doi.org/10.1590/S0104-11692007000300022

Souza, M. A. de. (2018). Analgesia para o parto vaginal: análise secundária do Estudo Multi-países da Organização Mundial de Saúde sobre Saúde Materna $e$ Neonatal. [Dissertação de Mestrado, Universidade Estadual de Campinas. Repositório da Produção Científica e Intelectual da Unicamp. http://repositorio.unicamp.br/bitstream/REPOSIP/333179/1/Souza_MarcioAntonioDe_D.pdf

Tostes, N. A., \& Seidl, E. M. F. (2016). Expectativas de gestantes sobre o parto e suas percepções acerca da preparação para o parto. Temas em Psicologia, 24(2), 681-693. https://doi.org/10.9788/TP2016.2-15

Trindade, D. F. S., Spinielli, M. A. dos S., \& Moreira, B. D. (2018). Modelos da comunicação no processo de humanização do parto e nascimento em uma maternidade de Mato Grosso, Brasil. Revista Brasileira de Pesquisa em Saúde, 20(2), 44-53.

United Nations. (2016). United Nations Development Programme.. http://www.undp.org/content/undp/en/home/sdgoverview/mdg_goals.html

Vidal, R. M. da S. (2017). A experiência do parto no Sistema Único de Saúde: Percepções de mulheres. https://repositorio.ufscar.br/handle/ufscar/9042

Weidle, W. G., Medeiros, C. R. G., Grave, M. T. Q., \& Dal Bosco, S. M. (2014). Escolha da via de parto pela mulher: Autonomia ou indução? Cadernos Saúde Coletiva, 22, 46-53. https://doi.org/10.1590/1414-462X201400010008

Workie, M. M., Chekol, W. B., Fentie, D. Y., Ahmed, S. A., \& Bizuneh, Y. B. (2021). Assessment of Awareness, Attitude and Desire for Labor Analgesia and Associated Factors Among Pregnant Women in Ethiopia: A Cross-Sectional Study. Pain and Therapy, 10(1), 363-376. https://doi.org/10.1007/s40122-020$00212-1$

Yadav, A., Karim, H. Md. R., Prakash, A., Jena, P., \& Aman, K. (2018). Correlation of different parity and school education with acceptance of labor analgesia among antenatal women: A questionnaire-based study. Saudi Journal of Anaesthesia, 12(2), 287-291. https://doi.org/10.4103/sja.SJA 474_17 